\title{
Singularities of orbit closures in module varieties and cones over rational normal curves
}

\author{
Grzegorz Zwara
}

July 31, 2018

\begin{abstract}
Let $N$ be a point of an orbit closure $\overline{\mathcal{O}}_{M}$ in a module variety such that its orbit $\mathcal{O}_{N}$ has codimension two in $\overline{\mathcal{O}}_{M}$. We show that under some additional conditions the pointed variety $\left(\overline{\mathcal{O}}_{M}, N\right)$ is smoothly equivalent to a cone over a rational normal curve.
\end{abstract}

\section{Introduction and the main results}

Throughout the paper, $k$ denotes an algebraically closed field, $A$ denotes a finitely generated associative $k$-algebra with identity, and by a module we mean a left $A$-module whose underlying vector space is finite dimensional. Let $d$ be a positive integer and denote by $\mathbb{M}(d)$ the algebra of $d \times d$-matrices with coefficients in $k$. The set $\bmod _{A}(d)$ of algebra homomorphisms $A \rightarrow \mathbb{M}(d)$ has a natural structure of an affine variety. Indeed, if $A \simeq k\left\langle X_{1}, \ldots, X_{t}\right\rangle / I$ for some two-sided ideal $I$, then $\bmod _{A}(d)$ can be identified with the closed subset of $(\mathbb{M}(d))^{t}$ given by vanishing of the entries of all matrices $\rho\left(X_{1}, \ldots, X_{t}\right), \rho \in$ $I$. Moreover, the general linear group $\mathrm{GL}(d)$ acts on $\bmod _{A}(d)$ by conjugations

$$
g \star\left(M_{1}, \ldots, M_{t}\right)=\left(g M_{1} g^{-1}, \ldots, g M_{t} g^{-1}\right),
$$

and the $\mathrm{GL}(d)$-orbits in $\bmod _{A}(d)$ correspond bijectively to the isomorphism classes of $d$-dimensional modules. We shall denote by $\mathcal{O}_{M}$ the $\mathrm{GL}(d)$-orbit

Mathematics Subject Classification (2000): 14L30 (Primary); 14B05, 16G10, 16G20 (Secondary).

Key Words and Phrases: Module varieties, orbit closures, types of singularities, rational normal curves. 
in $\bmod _{A}(d)$ corresponding to a $d$-dimensional module $M$. An interesting problem is to study geometric properties of the Zariski closure $\overline{\mathcal{O}}_{M}$ of an orbit $\mathcal{O}_{M}$ in $\bmod _{A}(d)$. We refer to [2], [3], [5], [6], 12, [13] and [14] for some results in this direction.

Following Hesselink (see [9, (1.7)]) we call two pointed varieties $\left(\mathcal{X}, x_{0}\right)$ and $\left(\mathcal{Y}, y_{0}\right)$ smoothly equivalent if there are smooth morphisms $f: \mathcal{Z} \rightarrow \mathcal{X}$, $g: \mathcal{Z} \rightarrow \mathcal{Y}$ and a point $z_{0} \in \mathcal{Z}$ with $f\left(z_{0}\right)=x_{0}$ and $g\left(z_{0}\right)=y_{0}$. This is an equivalence relation and the equivalence classes will be denoted by $\operatorname{Sing}\left(\mathcal{X}, x_{0}\right)$ and called the types of singularities. Obviously the regular points of the varieties form one type of singularity, which we denote by Reg. Let $M$ and $N$ be $d$-dimensional modules such that $N$ is a degeneration of $M$, i.e., $\mathcal{O}_{N} \subseteq \overline{\mathcal{O}}_{M}$. We shall write $\operatorname{Sing}(M, N)$ for $\operatorname{Sing}\left(\overline{\mathcal{O}}_{M}, n\right)$, where $n$ is an arbitrary point of $\mathcal{O}_{N}$. By [13, Thm.1.1], $\operatorname{Sing}(M, N)=\operatorname{Reg}$ provided $\operatorname{dim} \mathcal{O}_{M}-\operatorname{dim} \mathcal{O}_{N}=1$

Assume now that $\operatorname{dim} \mathcal{O}_{M}-\operatorname{dim} \mathcal{O}_{N}=2$. It was shown recently (14, Thm.1.3]) that $\operatorname{Sing}(M, N)=\operatorname{Reg}$ provided $A$ is the path algebra of a Dynkin quiver. However, other types of singularities occur if $A$ is the path algebra of the Kronecker quiver (see [2]); for instance, the type of an isolated singularity of the surface

$$
\mathcal{C}_{r}=\left\{\left(x_{0}, \ldots, x_{r}\right) \in k^{r+1} ; x_{i} x_{j}=x_{l} x_{m} \text { if } i+j=l+m\right\}, \quad r \geq 1 .
$$

The surface $\mathcal{C}_{r}$ is the affine cone over the rational normal curve (or Veronese curve) of degree $r$ (see [8] for an introduction to rational normal curves). Obviously $\operatorname{Sing}\left(\mathcal{C}_{r}, 0\right)=\operatorname{Reg}$ if and only if $r=1$. The main theorem of the paper shows that the above types of singularities occur quite frequently for orbit closures in module varieties. From now on, we abbreviate $\operatorname{dim}_{k} \operatorname{Hom}_{A}(U, V)$ by $[U, V]$ for any modules $U$ and $V$.

Theorem 1.1. Let $M$ and $N$ be modules such that $\operatorname{dim} \mathcal{O}_{M}-\operatorname{dim} \mathcal{O}_{N}=2$, $\mathcal{O}_{N} \subseteq \overline{\mathcal{O}}_{M}$ and $N=U \oplus V$ for some modules $U$ and $V$ satisfying

$$
[U, M]=[U, N] \quad \text { and } \quad[M, V]=[N, V] .
$$

Then $\operatorname{Sing}(M, N)=\operatorname{Sing}\left(\mathcal{C}_{r}, 0\right)$ for some $r \geq 1$.

As an application of this theorem we get the following result about preprojective modules (see Section $\mathbf{Z}$ for the definition).

Theorem 1.2. Assume that the algebra $A$ is finite dimensional. Let $M$ and $N$ be preprojective modules such that $\mathcal{O}_{N} \subseteq \overline{\mathcal{O}}_{M}$ and $\operatorname{dim} \mathcal{O}_{M}-\operatorname{dim} \mathcal{O}_{N}=2$. Then $\operatorname{Sing}(M, N)=\operatorname{Sing}\left(\mathcal{C}_{r}, 0\right)$ for some $r \geq 1$. 
One says that a module $N$ is a minimal degeneration of a module $M$ provided $\overline{\mathcal{O}}_{N} \varsubsetneqq \overline{\mathcal{O}}_{M}$ but there is no module $L$ with $\overline{\mathcal{O}}_{N} \varsubsetneqq \overline{\mathcal{O}}_{L} \varsubsetneqq \overline{\mathcal{O}}_{M}$. Moreover, two modules are called disjoint if they have no nonzero direct summand in common. Combining the main results in [4, [7] and [13] with Theorem 1.2, we get the following consequence.

Corollary 1.3. Let $A$ be the path algebra of an extended Dynkin quiver. Let $M$ and $N$ be two disjoint preprojective modules such that $N$ is a minimal degeneration of $M$. Then $\operatorname{Sing}(M, N)=\operatorname{Sing}\left(\mathcal{C}_{r}, 0\right)$ for some integer $r \geq 1$.

We consider in Section 2 some properties of short exact sequences, dimensions of homomorphism spaces and degenerations of modules. Sections 3 , 4 and 5 contain preliminary results necessary to prove Proposition [5.14 which plays a central role in the paper. Sections $\left[\begin{array}{l}\text { a } \\ \text { and }\end{array}\right]$ are devoted to the proofs of Theorems 1.1 and 1.2 respectively. In Section 8 we give an application of Theorem 1.1 different from the one described in Theorem 1.2

For a basic background on the representation theory of algebras and quivers we refer to [1] and 10. The author gratefully acknowledges support from the Polish Scientific Grant KBN No. 1 P03A 01827.

\section{Degenerations and extensions of modules}

Let $W$ be a vector space. We denote by $\mathbb{P} W$ the projective space of onedimensional subspaces in $W$. Thus we have the quotient map

$$
\pi: W \backslash\{0\} \rightarrow \mathbb{P} W, \quad w \mapsto k \cdot w,
$$

of $W \backslash\{0\}$ by $k^{*}$ acting by scalar multiplication. Moreover, we denote by $\mathbb{P} S$ the image $\pi(S \backslash\{0\})$ for any $k^{*}$-invariant subset $S$ of $W$.

Throughout the paper, we consider short exact sequences of modules

$$
\sigma: 0 \rightarrow U \stackrel{f}{\rightarrow} M \stackrel{g}{\rightarrow} V \rightarrow 0,
$$

where $U, M$ and $V$ are modules, $f$ is an $A$-module monomorphism, $g$ is an $A$ module epimorphism and $\operatorname{Im}(f)=\operatorname{Ker}(g)$. Any such sequence $\sigma$ determines an element of $\operatorname{Ext}_{A}^{1}(V, U)$ denoted by $[\sigma]$, such that $[\sigma]=0$ if and only if the sequence $\sigma$ splits, and $[\sigma]=[\tau]$ for another short exact sequence

$$
\tau: 0 \rightarrow U \stackrel{f^{\prime}}{\rightarrow} W \stackrel{g^{\prime}}{\rightarrow} V \rightarrow 0
$$


if and only if there is an $A$-isomorphism $h: M \rightarrow W$ making the following diagram commutative:

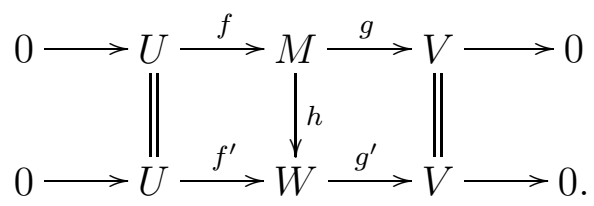

We denote by $\mathcal{E}_{M}(V, U)$ the subset of $\operatorname{Ext}_{A}^{1}(V, U)$ consisting of all elements $[\sigma]$, such that the middle term of the exact sequence $\sigma$ is isomorphic to $M$.

Lemma 2.1. $\mathcal{E}_{M}(V, U)$ is a locally closed $k^{*}$-invariant subset of the vector space $\operatorname{Ext}_{A}^{1}(V, U)$.

Proof. We use here an interpretation of the vector space $\operatorname{Ext}_{A}^{1}(V, U)$ as the quotient of the vector space $\mathbb{Z}_{A}^{1}(V, U)$ of cocycles, i.e., the $k$-linear maps $Z: A \rightarrow \operatorname{Hom}_{k}(V, U)$ satisfying

$$
Z\left(a a^{\prime}\right)=Z(a) V\left(a^{\prime}\right)+U(a) Z\left(a^{\prime}\right), \quad \text { for all } a, a^{\prime} \in A,
$$

by the subspace of coboundaries

$$
\mathbb{B}_{A}^{1}(V, U)=\left\{h V-U h ; h \in \operatorname{Hom}_{k}(V, U)\right\} .
$$

Let $d=\operatorname{dim}_{k}(U \oplus V)$. We take a subspace $\mathcal{W}$ of $\mathbb{Z}_{A}^{1}(V, U)$ complementary to $\mathbb{B}_{A}^{1}(V, U)$. Then the subset $\mathcal{W}^{\prime}$ of $\bmod _{A}(d)$ consisting of the algebra homomorphism of the block form $\left[\begin{array}{ll}U & Z \\ 0 & V\end{array}\right]$, where $Z \in \mathcal{W}$, is closed. Hence the claim follows from the fact that $\mathcal{E}_{M}(V, U)$ is isomorphic to the intersection $\mathcal{W}^{\prime} \cap \mathcal{O}_{M}$

The short exact sequence (2.1) induces two long exact sequences of vector spaces

$$
\begin{aligned}
0 \rightarrow \operatorname{Hom}_{A}(V, X) \stackrel{\operatorname{Hom}_{A}(g, X)}{\longrightarrow} \operatorname{Hom}_{A}(M, X) \stackrel{\operatorname{Hom}_{A}(f, X)}{\longrightarrow} \operatorname{Hom}_{A}(U, X) \stackrel{\partial}{\rightarrow} \\
\quad \stackrel{\partial}{\rightarrow} \operatorname{Ext}_{A}^{1}(V, X) \stackrel{\operatorname{Ext}_{A}^{1}(g, X)}{\longrightarrow} \operatorname{Ext}_{A}^{1}(M, X) \stackrel{\operatorname{Ext}_{A}^{1}(f, X)}{\longrightarrow} \operatorname{Ext}_{A}^{1}(U, X), \\
0 \rightarrow \operatorname{Hom}_{A}(X, U) \stackrel{\operatorname{Hom}_{A}(X, f)}{\longrightarrow} \operatorname{Hom}_{A}(X, M) \stackrel{\operatorname{Hom}_{A}(X, g)}{\longrightarrow} \operatorname{Hom}_{A}(X, V) \stackrel{\partial^{\prime}}{\rightarrow} \\
\stackrel{\partial^{\prime}}{\longrightarrow} \operatorname{Ext}_{A}^{1}(X, U) \stackrel{\operatorname{Ext}_{A}^{1}(X, f)}{\longrightarrow} \operatorname{Ext}_{A}^{1}(X, M) \stackrel{\operatorname{Ext}_{A}^{1}(X, g)}{\longrightarrow} \operatorname{Ext}_{A}^{1}(X, V),
\end{aligned}
$$


for any module $X$. Here,

$$
\partial(h)=\operatorname{Ext}_{A}^{1}(V, h)([\sigma]) \quad \text { and } \quad \partial^{\prime}\left(h^{\prime}\right)=\operatorname{Ext}_{A}^{1}\left(h^{\prime}, U\right)([\sigma])
$$

for any homomorphisms $h: U \rightarrow X$ and $h^{\prime}: X \rightarrow V$. These induced exact sequences suggest us to define the following two non-negative integers

$$
\begin{aligned}
& \delta_{\sigma}(X)=[U \oplus V, X]-[M, X]=\operatorname{dim}_{k} \operatorname{Im}(\partial) \geq 0 \\
& \delta_{\sigma}^{\prime}(X)=[X, U \oplus V]-[X, M]=\operatorname{dim}_{k} \operatorname{Im}\left(\partial^{\prime}\right) \geq 0
\end{aligned}
$$

Observe that the sequence $\sigma$ splits if and only if

$$
M \simeq U \oplus V \Longleftrightarrow \delta_{\sigma}(U)=0 \Longleftrightarrow \delta_{\sigma}^{\prime}(V)=0
$$

(see [14, Lem.2.3]). Taking the pushout of $\sigma$ under $h$ leads to the following commutative diagram with exact rows:

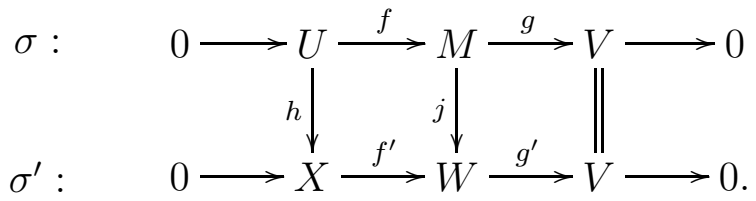

Then $\left[\sigma^{\prime}\right]=\operatorname{Ext}_{A}^{1}(V, h)([\sigma])=\partial(h)$. Moreover, the short sequence

$$
\tau: \quad 0 \rightarrow U \stackrel{\left(\begin{array}{l}
f \\
h
\end{array}\right)}{\longrightarrow} M \oplus X \stackrel{\left(j,-f^{\prime}\right)}{\longrightarrow} W \rightarrow 0
$$

is exact and

$$
\delta_{\sigma}(Y)=\delta_{\sigma^{\prime}}(Y)+\delta_{\tau}(Y), \quad \delta_{\sigma}^{\prime}(Y)=\delta_{\sigma^{\prime}}^{\prime}(Y)+\delta_{\tau}^{\prime}(Y),
$$

for any module $Y$. In particular, we obtain the following fact.

Corollary 2.2. Let $\sigma^{\prime}$ be a pushout of a short exact sequence $\sigma$. Then $\delta_{\sigma^{\prime}}(Y) \leq \delta_{\sigma}(Y)$ and $\delta_{\sigma^{\prime}}^{\prime}(Y) \leq \delta_{\sigma}^{\prime}(Y)$ for any module $Y$.

We shall need the following technical result.

Lemma 2.3. Let $\sigma: 0 \rightarrow U \oplus Y^{i} \stackrel{f}{\rightarrow} W \rightarrow V \rightarrow 0$ be a short exact sequence such that $\delta_{\sigma}(Y)<i$. Then $W$ is isomorphic to $W^{\prime} \oplus Y$ and there is a short exact sequence

$$
\sigma^{\prime}: 0 \rightarrow U \oplus Y^{i-1} \rightarrow W^{\prime} \rightarrow V \rightarrow 0
$$

for some module $W^{\prime}$. Moreover, $\delta_{\sigma^{\prime}}(X)=\delta_{\sigma}(X)$ for any module $X$. 
Proof. We consider the exact sequence

$$
0 \rightarrow \operatorname{Hom}_{A}(V, Y) \rightarrow \operatorname{Hom}_{A}(W, Y) \stackrel{\operatorname{Hom}_{A}(f, Y)}{\longrightarrow} \operatorname{Hom}_{A}\left(U \oplus Y^{i}, Y\right)
$$

induced by $\sigma$. Let $\mathcal{H}$ be the $i$-dimensional subspace of $\operatorname{Hom}_{A}\left(U \oplus Y^{i}, Y\right)$ spanned by $i$ canonical projections. Since the codimension of the image of $\operatorname{Hom}_{A}(f, Y)$ in $\operatorname{Hom}_{A}\left(U \oplus Y^{i}, Y\right)$ equals $\delta_{\sigma}(Y)<\operatorname{dim}_{k} \mathcal{H}$, the image contains a nonzero element $h \in \mathcal{H}$. Thus $h$ factors through $f$. We decompose

$$
f=\left(f_{0}, f_{1}, \ldots, f_{i}\right) \quad \text { and } \quad h=\left(0, \lambda_{1} \cdot 1_{Y}, \ldots, \lambda_{i} \cdot 1_{Y}\right) \neq 0 .
$$

Then $\lambda_{j} \neq 0$ and consequently $f_{j}$ is a section, for some $j \in\{1, \ldots, i\}$. The remaining part of the proof is now straightforward (for instance, use a dual version of [14, Lem.2.4]).

The next result ([1] ) gives a characterization of degenerations of modules.

Theorem 2.4. Let $M$ and $N$ be modules. Then the inclusion $\mathcal{O}_{N} \subseteq \overline{\mathcal{O}}_{M}$ is equivalent to each of the following conditions:

(1) There is a short exact sequence $0 \rightarrow Z \rightarrow Z \oplus M \rightarrow N \rightarrow 0$ for some module $Z$.

(2) There is a short exact sequence $0 \rightarrow N \rightarrow M \oplus Z^{\prime} \rightarrow Z^{\prime} \rightarrow 0$ for some module $Z^{\prime}$.

We shall need the following two well known direct consequences.

Corollary 2.5. Let $\sigma: 0 \rightarrow U \rightarrow M \rightarrow V \rightarrow 0$ be a short exact sequence. Then $\mathcal{O}_{U \oplus V} \subseteq \overline{\mathcal{O}}_{M}$.

Proof. We apply Theorem 2.4 to a direct sum of $\sigma$ and the short exact sequence $0 \rightarrow 0 \rightarrow U \stackrel{1_{U}}{\longrightarrow} U \rightarrow 0$.

Corollary 2.6. Let $M$ and $N$ be modules such that $\mathcal{O}_{N} \subseteq \overline{\mathcal{O}}_{M}$. Then

$$
[M, Y] \leq[N, Y] \quad \text { and } \quad[Y, M] \leq[Y, N]
$$

for any module $Y$.

Proof. Observe that

$$
\delta_{\sigma}(Y)=[N, Y]-[M, Y] \quad \text { and } \quad \delta_{\sigma}^{\prime}(Y)=[Y, N]-[Y, M]
$$

for any short exact sequence $\sigma$ of the form $0 \rightarrow Z \rightarrow Z \oplus M \rightarrow N \rightarrow 0$. 


\section{$3 \quad$ Generic quotients}

Throughout the section, $U, M$ and $V$ are modules such that there is a short exact sequence

$$
\sigma: 0 \rightarrow U \rightarrow M \rightarrow V \rightarrow 0
$$

with $\delta_{\sigma}(V)=0$. Let $d=\operatorname{dim}_{k} V$ and $\mathcal{Q}(U, M)$ be the subset of $\bmod _{A}(d)$ consisting of all points corresponding to quotients of $M$ by $U$. Obviously, the set $\mathcal{Q}(U, M)$ is $\mathrm{GL}(d)$-invariant. Moreover, it is constructible and irreducible, by [6, 2.3].

Lemma 3.1. The orbit $\mathcal{O}_{V}$ is an open subset of $\overline{\mathcal{Q}(U, M)}$.

Proof. Let $\mathcal{A}$ be the closed subset of $\overline{\mathcal{Q}(U, M)} \times \operatorname{Hom}_{k}\left(k^{d}, V\right)$ consisting of all pairs $\left(V^{\prime}, h\right)$ such that $h$ belongs to $\operatorname{Hom}_{A}\left(V^{\prime}, V\right)$. Then the projection $\pi: \mathcal{A} \rightarrow \overline{\mathcal{Q}(U, M)}$ is a surjective regular morphism. We know that $U \oplus V^{\prime}$ belongs to $\overline{\mathcal{O}}_{M}$ for any $V^{\prime} \in \mathcal{Q}(U, M)$, by Corollary 2.5. Hence the same holds for arbitrary $V^{\prime} \in \overline{\mathcal{Q}(U, M)}$. Consequently,

$$
\left[U \oplus V^{\prime}, V\right] \geq[M, V]=[U \oplus V, V] \quad \text { and } \quad\left[V^{\prime}, V\right] \geq[V, V]
$$

by Corollary 2.6 and since $\delta_{\sigma}(V)=0$. We consider the closed subset $\mathcal{A}^{\prime}$ of $\mathcal{A}$ consisting of the pairs $\left(V^{\prime}, h\right)$ such that $h$ is not invertible. Observe that

$$
\operatorname{dim}\left(\left.\pi\right|_{\mathcal{A}^{\prime}}\right)^{-1}\left(V^{\prime}\right)= \begin{cases}{[V, V]-1,} & \text { if } V^{\prime} \simeq V, \\ {\left[V^{\prime}, V\right],} & \text { if } V^{\prime} \nsucceq V .\end{cases}
$$

Hence $\left(\left.\pi\right|_{\mathcal{A}^{\prime}}\right)^{-1}\left(\mathcal{O}_{V}\right)$ is an open subset of $\mathcal{A}^{\prime}$. Applying the inverse image of the section

$$
\overline{\mathcal{Q}(U, M)} \rightarrow \mathcal{A}^{\prime}, \quad V^{\prime} \mapsto\left(V^{\prime}, 0\right),
$$

we get that $\mathcal{O}_{V}$ is an open subset of $\overline{\mathcal{Q}(U, M)}$.

Let $\mathcal{S}_{V}(U, M)$ denote the set of all $A$-monomorphisms $f: U \rightarrow M$ with $\operatorname{Coker}(f) \simeq V$.

Lemma 3.2. $\mathcal{S}_{V}(U, M)$ is an open subset of $\operatorname{Hom}_{A}(U, M)$.

Proof. We use a construction described in [5, 2.1]. Let $\mathcal{G}$ be the subset of $\operatorname{Hom}_{A}(U, M) \times \operatorname{Hom}_{k}\left(k^{d}, M\right)$ consisting of pairs $g=\left(g_{1}, g_{2}\right)$ such that the map $g: U \oplus k^{d} \rightarrow M$ is invertible. Then the projection $\pi: \mathcal{G} \rightarrow \operatorname{Hom}_{A}(U, M)$ is a composition of an open immersion followed by a trivial vector bundle. Moreover, if $g \in \mathcal{G}$ then $g^{-1} \star M$ has a block form

$$
\left[\begin{array}{cc}
U & Z \\
0 & V^{\prime}
\end{array}\right]
$$


where $V^{\prime}$ belongs to the set $\mathcal{Q}(U, M)$. This leads to a regular morphism of varieties

$$
\theta: \mathcal{G} \rightarrow \overline{\mathcal{Q}(U, M)}, \quad g \mapsto V^{\prime} .
$$

Then $\theta^{-1}\left(\mathcal{O}_{V}\right)$ is open in $\mathcal{G}$, by Lemma 3.1. Since the map $\pi$ is open, the set $\mathcal{S}_{V}(U, M)=\pi\left(\theta^{-1}\left(\mathcal{O}_{V}\right)\right)$ is open in $\operatorname{Hom}_{A}(U, M)$.

\section{Monomorphisms and extensions}

Throughout the section, $U, M$ and $V$ are modules such that there is a short exact sequence

$$
\sigma: 0 \rightarrow U \stackrel{f}{\rightarrow} M \rightarrow V \rightarrow 0
$$

with $\delta_{\sigma}(V)=0$ and $\delta_{\sigma}^{\prime}(V)=1$.

Lemma 4.1. Let $\tau: 0 \rightarrow U \stackrel{f^{\prime}}{\rightarrow} W \rightarrow V \rightarrow 0$ be a nonsplittable short exact sequences such that $f$ factors through $f^{\prime}$ (equivalently, the pushout of $\tau$ under $f$ is a splittable sequence). Then $W \simeq M$ and the equality $k \cdot[\tau]=k \cdot[\sigma]$ holds in $\mathbb{P} \operatorname{Ext}_{A}^{1}(V, U)$.

Proof. Since $f$ factors through $f^{\prime}$, we get the following commutative diagram

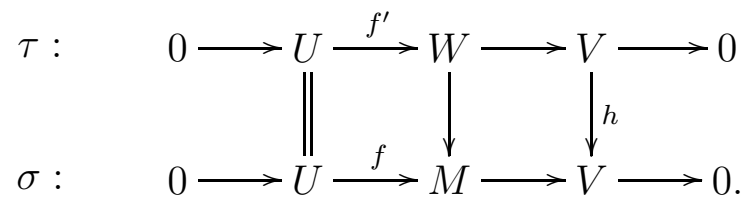

We consider the long exact sequence

$$
0 \rightarrow \operatorname{Hom}_{A}(V, U) \rightarrow \operatorname{Hom}_{A}(V, M) \rightarrow \operatorname{Hom}_{A}(V, V) \stackrel{\partial}{\rightarrow} \operatorname{Ext}_{A}^{1}(V, U)
$$

induced by $\sigma$. Then $\partial\left(1_{V}\right)=[\sigma]$ and $\partial(h)=[\tau]$. Now the claim follows from the equalities $1=\delta_{\sigma}^{\prime}(V)=\operatorname{dim}_{k} \operatorname{Im}(\partial)$ and since $[\sigma] \neq 0 \neq[\tau]$.

The above lemma shows the existence of a unique surjective map

$$
\xi_{U, M, V}: \mathcal{S}_{V}(U, M) \rightarrow \mathbb{P} \mathcal{E}_{M}(V, U)
$$

such that $\xi_{U, M, V}\left(f^{\prime}\right)=k \cdot\left[\sigma^{\prime}\right]$ for any short exact sequence of the form

$$
\sigma^{\prime}: 0 \rightarrow U \stackrel{f^{\prime}}{\rightarrow} M \rightarrow V \rightarrow 0 .
$$

Lemma 4.2. $\xi_{U, M, V}$ is a regular morphism of varieties. 
Proof. Let $d=\operatorname{dim}_{k} V$. We introduce three new varieties and four regular morphisms:

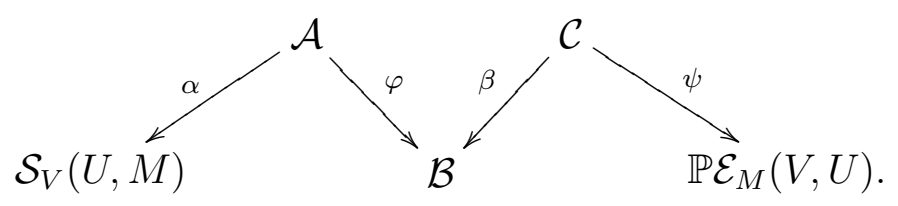

Here $\mathcal{A}$ is the subset of $\mathcal{S}_{V}(U, M) \times \operatorname{Hom}_{k}\left(k^{d}, M\right)$ consisting of all pairs $g=\left(g_{1}, g_{2}\right)$ such that the map $g: U \oplus k^{d} \rightarrow M$ is invertible; $\mathcal{B}$ is the subset of $\mathcal{O}_{M}$ consisting of all elements having a block form

$$
\left[\begin{array}{cc}
U & Z \\
0 & W
\end{array}\right]
$$

with $W \in \mathcal{O}_{V}$; and $\mathcal{C}$ is the subset of $\mathcal{B} \times \operatorname{Hom}_{k}\left(V, k^{d}\right)$ consisting of all pairs $\left(\left[\begin{array}{cc}U & Z \\ 0 & W\end{array}\right], h\right)$ such that $h$ is an isomorphism in $\operatorname{Hom}_{A}(V, W)$. Moreover, $\alpha$ and $\beta$ are obvious projections, and $\varphi(g)=g^{-1} \star M$. If $c=\left(\left[\begin{array}{cc}U & Z \\ 0 & W\end{array}\right], h\right)$ belongs to $\mathcal{C}$, then

$$
\left[\begin{array}{cc}
1_{U} & 0 \\
0 & h^{-1}
\end{array}\right] \star\left[\begin{array}{cc}
U & Z \\
0 & W
\end{array}\right]=\left[\begin{array}{cc}
U & Z h \\
0 & V
\end{array}\right] \in \mathcal{O}_{M},
$$

where $Z h$ belongs to $\mathbb{Z}_{A}^{1}(V, U) \backslash \mathbb{B}_{A}^{1}(V, U)$. We define $\psi(c)$ as the image of $Z h$ via the following composition of canonical quotients

$$
\mathbb{Z}_{A}^{1}(V, U) \backslash \mathbb{B}_{A}^{1}(V, U) \rightarrow \operatorname{Ext}_{A}^{1}(V, U) \backslash\{0\} \rightarrow \mathbb{P E x t}_{A}^{1}(V, U) .
$$

One can find that $\alpha$ and $\beta$ are surjective maps being compositions of open immersions followed by vector bundles (see for instance [5, 2.1]). Hence $\alpha$ and $\beta$ have enough local sections, i.e., there are open neighbourhoods $\mathcal{U}$ of $s$ and $\mathcal{V}$ of $b$ together with regular morphisms (sections) $\alpha^{\prime}: \mathcal{U} \rightarrow \mathcal{A}, \beta^{\prime}: \mathcal{V} \rightarrow \mathcal{C}$ such that $\alpha \alpha^{\prime}=1_{\mathcal{U}}$ and $\beta \beta^{\prime}=1_{\mathcal{V}}$, for any $s \in \mathcal{S}_{V}(U, M)$ and $b \in \mathcal{B}$.

We take $s \in \mathcal{S}_{V}(U, M)$ and consider a local section $\alpha^{\prime}: \mathcal{U} \rightarrow \mathcal{A}$ of $\alpha$ with $\mathcal{U}$ containing $s$. Let $a=\alpha^{\prime}(s)$ and $b=\varphi(a)$. We take also a neighbourhood $\mathcal{V}$ of $b$ and a local section $\beta^{\prime}: \mathcal{V} \rightarrow \mathcal{C}$ of $\beta$. Then $\mathcal{U}^{\prime}=\left(\varphi \alpha^{\prime}\right)^{-1}(\mathcal{V})$ is an open neighbourhood of $s$ and it follows from our constructions that $\xi_{U, M, V}\left(s^{\prime}\right)=$ $\psi \beta^{\prime} \varphi \alpha^{\prime}\left(s^{\prime}\right)$ for any $s^{\prime} \in \mathcal{U}^{\prime}$. Therefore $\left.\xi_{U, M, V}\right|_{\mathcal{U}^{\prime}}$ is a regular morphism.

The set $\mathcal{S}_{V}(U, M)$ is irreducible, by Lemma 3.2. Applying the surjective morphism $\xi_{U, M, V}$ we get the following consequence.

Corollary 4.3. $\mathbb{P} \mathcal{E}_{M}(V, U)$ is an irreducible variety. 


\section{$5 \quad$ Extensions and rational normal curves}

Throughout the section,

$$
\sigma_{1}: 0 \rightarrow U_{0} \stackrel{f_{1}}{\rightarrow} U_{1} \stackrel{h_{1}}{\rightarrow} V \rightarrow 0
$$

is a short exact sequence such that

$$
\delta_{\sigma_{1}}\left(U_{1}\right)=1, \quad \delta_{\sigma_{1}}(V)=0 \quad \text { and } \quad \delta_{\sigma_{1}}^{\prime}(V)=1 .
$$

Our aim is to prove that $\mathbb{P} \overline{\mathcal{E}_{U_{1}}\left(V, U_{0}\right)}$ is a rational normal curve contained in $\mathbb{P} \operatorname{Ext}_{A}^{1}\left(V, U_{0}\right)$.

We consider the exact sequence

$$
0 \rightarrow \operatorname{Hom}_{A}\left(V, U_{1}\right) \rightarrow \operatorname{Hom}_{A}\left(U_{1}, U_{1}\right) \stackrel{\operatorname{Hom}_{A}\left(f_{1}, U_{1}\right)}{\longrightarrow} \operatorname{Hom}_{A}\left(U_{0}, U_{1}\right)
$$

induced by $\sigma_{1}$. Since $\delta_{\sigma_{1}}\left(U_{1}\right)>0$ then there is an $A$-homomorphism $g_{1}: U_{0} \rightarrow$ $U_{1}$ not belonging to the image of $\operatorname{Hom}_{A}\left(f_{1}, U_{1}\right)$. We construct successive pushouts to obtain the following commutative diagram with exact rows

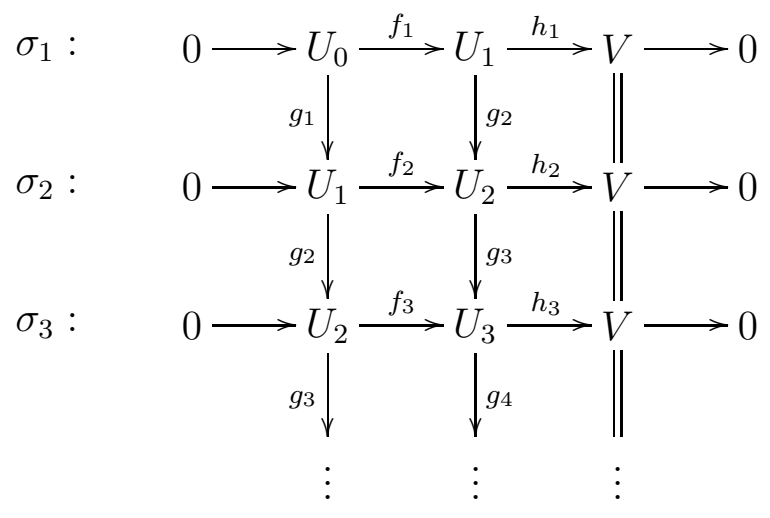

Applying Corollary 2.2 we get the following fact.

Corollary 5.1. $\delta_{\sigma_{i}}(V)=0$ and $\delta_{\sigma_{i}}^{\prime}(V) \leq 1$ for any $i \geq 1$.

Lemma 5.2. There is a short exact sequence

$$
0 \rightarrow U_{0} \rightarrow U_{i} \rightarrow V^{i} \rightarrow 0
$$

for any $i \geq 1$.

Proof. We proceed by induction on $i$. Assume that there is a short exact sequence

$$
0 \rightarrow U_{0} \stackrel{f^{\prime}}{\rightarrow} U_{i} \stackrel{h^{\prime}}{\rightarrow} V^{i} \rightarrow 0
$$


for some $i \geq 1$. Taking the pushout of $\sigma_{i+1}$ under $h^{\prime}$ we obtain the following commutative diagram with exact rows and columns

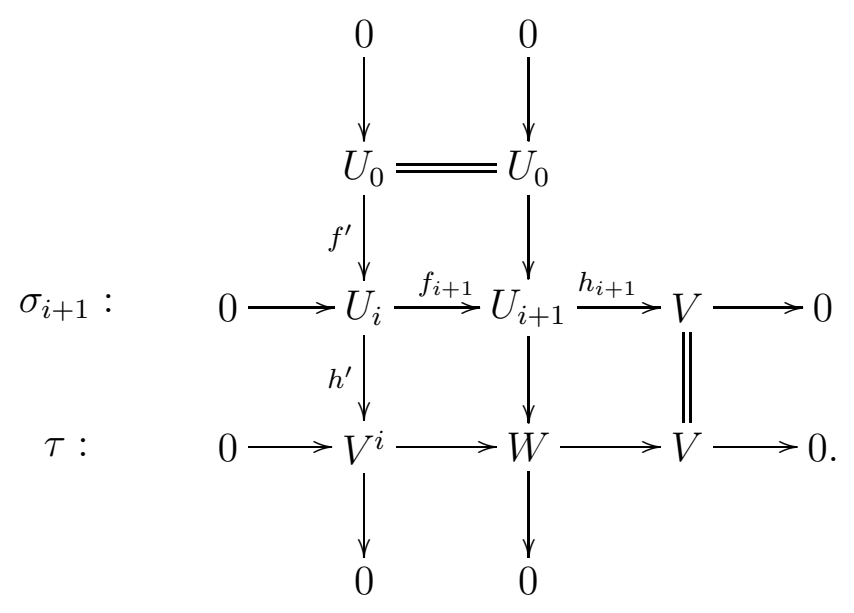

Thus it suffices to show that $W$ is isomorphic to $V^{i+1}$. Applying Corollaries 2.2 and 5.1, we get $\delta_{\tau}(V) \leq \delta_{\sigma_{i+1}}(V)=0$. Hence $\delta_{\tau}\left(V^{i}\right)=0$. Therefore the short exact sequence $\tau$ splits and $W$ is isomorphic to $V^{i} \oplus V$.

Lemma 5.3. The short exact sequence $\sigma_{i}$ splits for some $i$.

Proof. Let $i$ be an integer not smaller than $j=\operatorname{dim}_{k} \operatorname{Ext}_{A}^{1}\left(V, U_{0}\right)$. We get from Lemma 5.2 a short exact sequence of the form

$$
\tau: 0 \rightarrow U_{0} \rightarrow U_{i} \rightarrow V^{i} \rightarrow 0
$$

It follows from the long exact sequence

$$
0 \rightarrow \operatorname{Hom}_{A}\left(V, U_{0}\right) \rightarrow \operatorname{Hom}_{A}\left(V, U_{i}\right) \rightarrow \operatorname{Hom}_{A}\left(V, V^{i}\right) \rightarrow \operatorname{Ext}_{A}^{1}\left(V, U_{0}\right)
$$

induced by $\tau$ that $\delta_{\tau}^{\prime}(V) \leq j$. Applying $(i-j)$ times a dual version of Lemma 2.3 for the sequence $\tau$ we get that $U_{i} \simeq V^{i-j} \oplus \widetilde{U}_{i}$ for some module $\widetilde{U}_{i}$ of dimension

$$
d=\operatorname{dim}_{k} U_{0}+j \cdot \operatorname{dim}_{k} V .
$$

Hence the sequence $\sigma_{i+1}$ has the form

$$
0 \rightarrow V^{i-j} \oplus \widetilde{U}_{i} \rightarrow V^{i+1-j} \oplus \widetilde{U}_{i+1} \rightarrow V \rightarrow 0 .
$$

We know that $\delta_{\sigma_{i+1}}(V)=0$, by Corollary 5.1. Then applying $(i-j)$ times Lemma 2.3 we get an exact sequence of the form

$$
0 \rightarrow \widetilde{U}_{i} \rightarrow \widetilde{U}_{i+1} \oplus V \rightarrow V \rightarrow 0
$$


Thus $\mathcal{O}_{\widetilde{U}_{i}} \subseteq \overline{\mathcal{O}}_{\widetilde{U}_{i+1}}$, by Theorem 2.4. Obviously the chain of inclusions

$$
\overline{\mathcal{O}}_{\widetilde{U}_{j}} \subseteq \overline{\mathcal{O}}_{\widetilde{U}_{j+1}} \subseteq \overline{\mathcal{O}}_{\widetilde{U}_{j+2}} \subseteq \ldots
$$

between orbit closures in $\bmod _{A}(d)$ must terminate, i.e., $\overline{\mathcal{O}}_{\widetilde{U}_{l}}=\overline{\mathcal{O}}_{\widetilde{U}_{l+1}}$ for some $l>j$. Hence $\widetilde{U}_{l}$ is isomorphic to $\widetilde{U}_{l+1}$ and the sequence $\sigma_{l+1}$ splits.

By our assumptions the sequence $\sigma_{1}$ does not split. Moreover, the same holds for $\sigma_{2}$, by our choice of $g_{1}$. Let $n \geq 1$ be the smallest integer such that the sequence $\sigma_{n+2}$ splits. As a direct consequence of Corollary 5.1 we get the following fact.

Corollary 5.4. $\delta_{\sigma_{i}}(V)=0$ and $\delta_{\sigma_{i}}^{\prime}(V)=1$ for $i \in\{1,2, \ldots, n+1\}$.

Let $i \in\{1,2, \ldots, n\}$. Since the sequence $\sigma_{i+1}$ does not split, $g_{i}$ does not factor through the monomorphism $f_{i}$. Consequently, the subspace $\mathcal{H}_{i}$ of $\operatorname{Hom}_{A}\left(U_{i}, U_{i+1}\right)$ spanned by $f_{i}$ and $g_{i}$ has dimension 2. The bilinear map

$$
F_{i}: \operatorname{Ext}_{A}^{1}\left(V, U_{i-1}\right) \times \operatorname{Hom}_{A}\left(U_{i-1}, U_{i}\right) \rightarrow \operatorname{Ext}_{A}^{1}\left(V, U_{i}\right),
$$

defined by $F_{i}(e, f)=\operatorname{Ext}_{A}^{1}(V, f)(e)$, induces a linear map

$$
F_{i}^{\prime}: \operatorname{Ext}_{A}^{1}\left(V, U_{i-1}\right) \rightarrow \operatorname{Hom}_{k}\left(\mathcal{H}_{i}, \operatorname{Ext}_{A}^{1}\left(V, U_{i}\right)\right),
$$

such that $\left(F_{i}^{\prime}(e)\right)(f)=F_{i}(e, f)=\operatorname{Ext}_{A}^{1}(V, f)(e)$.

Lemma 5.5. The linear map $F_{i}^{\prime}$ is injective for $i \leq n$.

Proof. Let $\sigma: 0 \rightarrow U_{i-1} \rightarrow W \rightarrow V \rightarrow 0$ be a short exact sequence such that $F_{i}^{\prime}([\sigma])=0$. Then the pushout of $\sigma$ under $f_{i}$ splits, and consequently, $[\sigma]=c \cdot\left[\sigma_{i}\right]$ for some scalar $c \in k$, by Lemma 4.1] and Corollary 5.4. We also know that the pushout of $\sigma$ under $g_{i}$ splits, which means that

$$
0=\operatorname{Ext}_{A}^{1}\left(V, g_{i}\right)([\sigma])=c \cdot \operatorname{Ext}_{A}^{1}\left(V, g_{i}\right)\left(\left[\sigma_{i}\right]\right)=c \cdot\left[\sigma_{i+1}\right] .
$$

By our construction, $\left[\sigma_{i+1}\right] \neq 0$. Thus $c=0$ and $[\sigma]=0$.

Let $W$ and $W^{\prime}$ be two vector spaces. If at least one of them is finite dimensional then the monomorphism

$$
F: W^{*} \otimes_{k} W^{\prime} \rightarrow \operatorname{Hom}_{k}\left(W, W^{\prime}\right), \quad F\left(\xi \otimes w^{\prime}\right)(w)=\xi(w) \cdot w^{\prime},
$$

is an isomorphism. Here $W^{*}$ denotes the dual space of $W$. Thus $F_{i}^{\prime}$ induces an injective linear map

$$
F_{i}^{\prime \prime}: \operatorname{Ext}_{A}^{1}\left(V, U_{i-1}\right) \rightarrow \mathcal{H}_{i}^{*} \otimes \operatorname{Ext}_{A}^{1}\left(V, U_{i}\right)
$$

for $i \in\{1, \ldots, n\}$. Combining these maps we get an injective linear map

$$
F^{\prime \prime}: \operatorname{Ext}_{A}^{1}\left(V, U_{0}\right) \rightarrow \mathcal{H}_{1}^{*} \otimes \mathcal{H}_{2}^{*} \otimes \cdots \otimes \mathcal{H}_{n}^{*} \otimes \operatorname{Ext}_{A}^{1}\left(V, U_{n}\right) .
$$


Lemma 5.6. $\operatorname{rk}\left(F_{1}^{\prime}(e)\right)=1$ for any $e \in \overline{\mathcal{E}_{U_{1}}\left(V, U_{0}\right)} \backslash\{0\}$.

Proof. We know that $\operatorname{rk}\left(F_{1}^{\prime}(e)\right) \geq 1$, by Lemma 5.5. Assume now that $e$ belongs to $\mathcal{E}_{U_{1}}\left(V, U_{0}\right)$. It suffices to show that $\operatorname{rk}\left(F_{1}^{\prime}(e)\right) \leq 1$, as the function $\operatorname{rk}\left(F_{1}^{\prime}(-)\right): \operatorname{Ext}_{A}^{1}\left(V, U_{0}\right) \rightarrow \mathbb{Z}$ is semicontinuous. We take a short exact sequence

$$
\sigma: 0 \rightarrow U_{0} \rightarrow U_{1} \rightarrow V \rightarrow 0
$$

such that $[\sigma]=e$. Since $\delta_{\sigma}\left(U_{1}\right)=1$, the kernel of the last map $\partial=$ $\operatorname{Ext}_{A}^{1}(V,-)([\sigma])$ in the long exact sequence

$$
0 \rightarrow \operatorname{Hom}_{A}\left(V, U_{1}\right) \rightarrow \operatorname{Hom}_{A}\left(U_{1}, U_{1}\right) \rightarrow \operatorname{Hom}_{A}\left(U_{0}, U_{1}\right) \stackrel{\partial}{\rightarrow} \operatorname{Ext}_{A}^{1}\left(V, U_{1}\right)
$$

induced by $\sigma$ has codimension 1 . We know that $\operatorname{dim} \mathcal{H}_{1}=2$ and therefore there is a nonzero homomorphism $h \in \mathcal{H}_{1}$ such that

$$
0=\partial(h)=\operatorname{Ext}_{A}^{1}(V, h)([\sigma])=F_{1}^{\prime}([\sigma])(h) .
$$

Consequently, $F_{1}^{\prime}(e)$ is not injective and $\operatorname{rk}\left(F_{1}^{\prime}(e)\right) \leq 1$.

If $e \in \overline{\mathcal{E}_{U_{1}}\left(V, U_{0}\right)} \backslash\{0\}$ then $\operatorname{Ker}\left(F_{1}^{\prime}(e)\right)$ has dimension 1. Moreover,

$$
\operatorname{Ker}\left(F_{1}^{\prime}(\lambda \cdot e)\right)=\operatorname{Ker}\left(\lambda \cdot F_{1}^{\prime}(e)\right)=\operatorname{Ker}\left(F_{1}^{\prime}(e)\right)
$$

for any nonzero scalar $\lambda \in k$. Thus we obtain a regular morphism

$$
\varphi_{1}: \mathbb{P} \overline{\mathcal{E}_{U_{1}}\left(V, U_{0}\right)} \rightarrow \mathbb{P} \mathcal{H}_{1}, \quad \varphi_{1}(k \cdot e)=\operatorname{Ker}\left(F_{1}^{\prime}(e)\right) .
$$

Let $\mathcal{H}_{1}^{\diamond}=\mathcal{H}_{1} \cap \mathcal{S}_{V}\left(U_{0}, U_{1}\right)$. It is an open, dense and $k^{*}$-invariant subset of $\mathcal{H}_{1}$, by Lemma 3.2. We can consider a regular map

$$
\widehat{\varphi}_{1}: \mathbb{P} \mathcal{H}_{1}^{\diamond} \rightarrow \mathbb{P} \mathcal{E}_{U_{1}}\left(V, U_{0}\right), \quad \widehat{\varphi}_{1}(k \cdot h)=\xi_{U_{0}, U_{1}, V}(h) .
$$

Lemma 5.7. $\varphi_{1} \circ \widehat{\varphi}_{1}(k \cdot h)=k \cdot h$ for any $h \in \mathcal{H}_{1}^{\diamond}$.

Proof. Let $h \in \mathcal{H}_{1}^{\diamond}$. Then there is a short exact sequence of the form

$$
\sigma: 0 \rightarrow U_{0} \stackrel{h}{\rightarrow} U_{1} \rightarrow V \rightarrow 0 .
$$

Obviously, $\widehat{\varphi}_{1}(k \cdot h)=k \cdot[\sigma]$. Since the pushout of $\sigma$ under $h$ splits, $h$ belongs to the kernel of $F_{1}^{\prime}([\sigma])$. But the kernel has dimension 1 and therefore

$$
k \cdot h=\operatorname{Ker}\left(F_{1}^{\prime}([\sigma])\right)=\varphi_{1}(k \cdot[\sigma])=\varphi_{1}\left(\widehat{\varphi}_{1}(k \cdot h)\right) .
$$


Lemma 5.8. $\varphi_{1}^{-1}(k \cdot h)=\left\{\widehat{\varphi}_{1}(k \cdot h)\right\}$ for any $h \in \mathcal{H}_{1}^{\diamond}$.

Proof. Since $h \in \mathcal{S}_{V}\left(U_{0}, U_{1}\right)$, there is a short exact sequence of the form

$$
\tau: 0 \rightarrow U_{0} \stackrel{h}{\rightarrow} U_{1} \rightarrow V \rightarrow 0 .
$$

Let $\sigma: 0 \rightarrow U_{0} \stackrel{f^{\prime}}{\rightarrow} W \rightarrow V \rightarrow 0$ be a short exact sequence such that $[\sigma] \in \overline{\mathcal{E}_{U_{1}}\left(V, U_{0}\right)} \backslash\{0\}$ and $\varphi_{1}(k \cdot[\sigma])=k \cdot h$. We have to show that $k \cdot[\sigma]=$ $\xi_{U_{0}, U_{1}, V}(h)$. Since $h$ belongs to the kernel of $F_{i}^{\prime}([\sigma]), h$ factors through $f^{\prime}$. Hence $k \cdot[\sigma]=k \cdot[\tau]=\xi_{U_{0}, U_{1}, V}(h)$, by Lemma 4.1 and the equalities

$$
\delta_{\tau}(V)=\delta_{\sigma_{1}}(V)=0 \quad \text { and } \quad \delta_{\tau}^{\prime}(V)=\delta_{\sigma_{1}}^{\prime}(V)=1 .
$$

Lemma 5.9. The map $\widehat{\varphi}_{1}: \mathbb{P} \mathcal{H}_{1}^{\diamond} \rightarrow \mathbb{P} \mathcal{E}_{U_{1}}\left(V, U_{0}\right)$ is dominant and the map $\varphi_{1}: \mathbb{P} \overline{\mathcal{E}_{U_{1}}\left(V, U_{0}\right)} \rightarrow \mathbb{P} \mathcal{H}_{1}$ is an isomorphism.

Proof. Lemma 5.8 implies that $\widehat{\varphi}_{1}\left(\mathbb{P} \mathcal{H}_{1}^{\diamond}\right)=\varphi_{1}^{-1}\left(\mathbb{P} \mathcal{H}_{1}^{\diamond}\right)$ is an open subset of $\mathbb{P} \overline{\mathcal{E}_{U_{1}}\left(V, U_{0}\right)}$. Moreover, $\mathbb{P} \overline{\mathcal{E}_{U_{1}}\left(V, U_{0}\right)}$ is irreducible, by Corollary 4.3 . Hence the map $\widehat{\varphi}_{1}$ is dominant and $\varphi_{1}$ is birational. Finally, $\varphi_{1}$ is an isomorphism as it is a projective map and $\mathbb{P} \mathcal{H}_{1}$ is a smooth curve.

We define inductively subsets $\mathcal{E}_{i} \subseteq \operatorname{Ext}_{A}^{1}\left(V, U_{i-1}\right)$ for $i \in\{1, \ldots, n+1\}$ as follows:

$$
\mathcal{E}_{1}=\overline{\mathcal{E}_{U_{1}}\left(V, U_{0}\right)}, \quad \mathcal{E}_{i+1}=F_{i}\left(\mathcal{E}_{i} \times \mathcal{H}_{i}\right) .
$$

We consider the cofinite subset $\Lambda$ of $k$ consisting of the scalars $\lambda$ such that $f_{1}+\lambda \cdot g_{1}$ belongs to $\mathcal{S}_{V}\left(U_{0}, U_{1}\right)$, or equivalently to $\mathcal{H}_{1}^{\diamond}$. If $\lambda \in \Lambda$ then we derive from the diagram (5.1) the following commutative diagram with exact rows:

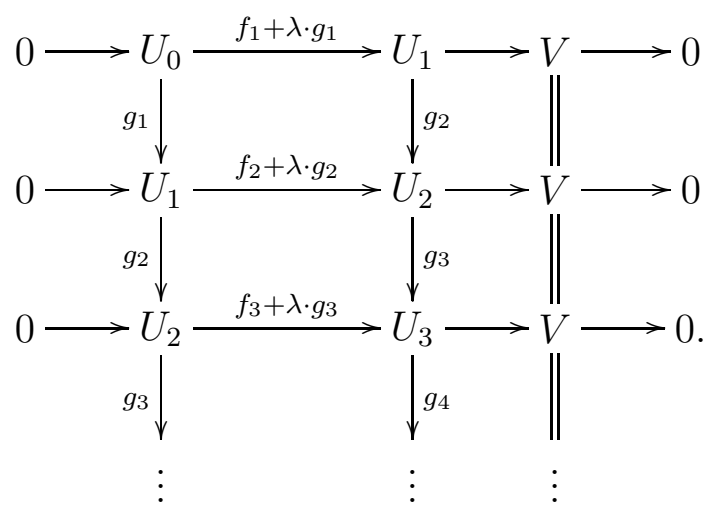

This implies the following fact. 
Corollary 5.10. The homomorphism $f_{i}+\lambda \cdot g_{i}$ belongs to $\mathcal{S}_{V}\left(U_{i-1}, U_{i}\right)$ for any $\lambda \in \Lambda$ and $i \geq 1$.

We derive from Corollaries 5.4 and 5.10 a regular map

$$
\eta_{i}: \Lambda \rightarrow \mathbb{P} \mathcal{E}_{i}, \quad \eta_{i}(\lambda)=\xi_{U_{i-1}, U_{i}, V}\left(f_{i}+\lambda \cdot g_{i}\right),
$$

for any $i \in\{1,2, \ldots, n+1\}$.

Lemma 5.11. Let $i \in\{1,2, \ldots, n+1\}$. Then:

(1) The map $\eta_{i}: \Lambda \rightarrow \mathbb{P} \mathcal{E}_{i}$ is dominant.

(2) $\operatorname{rk}\left(F_{i}^{\prime}(e)\right)=1$ provided $i \leq n$ and $e \in \mathcal{E}_{i} \backslash\{0\}$.

Proof. We proceed by induction on $i$. For $i=1$ the claim follows from Lemmas 5.6 and 5.9. Assume now that the claim holds for some $i \leq n$. By (2), we can consider two regular maps

$$
\begin{array}{rlrl}
\varphi_{i} & : \mathbb{P} \mathcal{E}_{i} \rightarrow \mathbb{P} \mathcal{H}_{i}, & \varphi_{i}(k \cdot e) & =\operatorname{Ker}\left(F_{i}^{\prime}(e)\right), \\
\psi_{i}: \mathbb{P} \mathcal{E}_{i} \rightarrow \mathbb{P} \mathcal{E}_{i+1}, & \psi_{i}(k \cdot e) & =\operatorname{Im}\left(F_{i}^{\prime}(e)\right) .
\end{array}
$$

Moreover, $\psi_{i}$ is a surjective map such that

$$
\psi_{i}\left(\eta_{i}(\lambda)\right)=\eta_{i+1}(\lambda), \quad \lambda \in \Lambda,
$$

by the diagram (5.2). Thus $\eta_{i+1}$ is a dominant map. Assume now that $i<n$. Observe that

$$
\mathcal{E}_{i+1}^{\diamond}=\left\{e \in \mathcal{E}_{i+1} \backslash\{0\} ; k \cdot e \in \operatorname{Im}\left(\eta_{i+1}\right)\right\}
$$

is a dense subset of $\mathcal{E}_{i+1}$. Hence by Lemma [5.5, it suffices to show that $\operatorname{rk}\left(F_{i+1}^{\prime}(e)\right) \leq 1$ for any $e \in \mathcal{E}_{i+1}^{\diamond}$. Let $e \in \mathcal{E}_{i+1}^{\diamond}$. Then there is a short exact sequence of the form

$$
\tau: 0 \rightarrow U_{i} \stackrel{f_{i+1}+\lambda \cdot g_{i+1}}{\longrightarrow} U_{i+1} \rightarrow V \rightarrow 0
$$

with $[\tau]=e$, for some $\lambda \in \Lambda$. Consequently $F_{i+1}^{\prime}(e)\left(f_{i+1}+\lambda \cdot g_{i+1}\right)=0$ and $F_{i+1}^{\prime}(e)$ is not an injective map. $\operatorname{Thus} \operatorname{rk}\left(F_{i+1}^{\prime}(e)\right)<\operatorname{dim}_{k} \mathcal{H}_{i+1}=2$.

We get from the above proof the following regular maps:

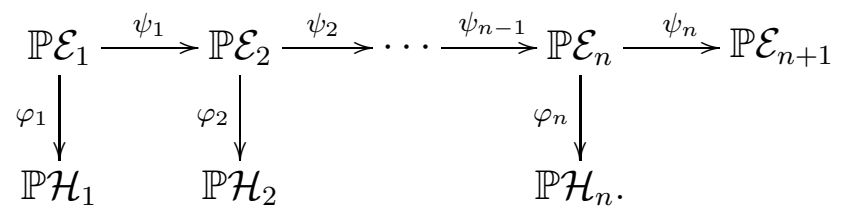


Lemma 5.12. The map $\mu_{i}=\varphi_{i} \psi_{i-1} \ldots \psi_{1}: \mathbb{P} \mathcal{E}_{1} \rightarrow \mathbb{P} \mathcal{H}_{i}$ is an isomorphism for $i \in\{1,2, \ldots, n\}$.

Proof. We consider the map $\eta=\mu_{i} \circ \varphi_{1}^{-1}: \mathbb{P} \mathcal{H}_{1} \rightarrow \mathbb{P} \mathcal{H}_{i}$ between projective lines. It follows from the diagram (5.2) that $\eta\left(k \cdot\left(f_{1}+\lambda g_{1}\right)\right)=k \cdot\left(f_{i}+\lambda g_{i}\right)$ for any $\lambda \in \Lambda$. Hence $\eta$ and $\eta \varphi_{1}=\mu_{i}$ are isomorphisms of varieties.

Lemma 5.13. $\mathbb{P} \mathcal{E}_{n+1}=k \cdot\left[\sigma_{n+1}\right]$.

Proof. Since the sequence $\sigma_{n+2}$ splits, the homomorphism $g_{n+1}$ factors through $f_{n+1}$. By Lemma 5.11(1) applied for $i=n+1$, it suffices to show that $\xi_{U_{n}, U_{n+1}, V}\left(f_{n+1}+\lambda g_{n+1}\right)=k \cdot\left[\sigma_{n+1}\right]$ for any $\lambda \in \Lambda$. The latter is true by Lemma 4.1. Corollary 5.4 and since $f_{n+1}+\lambda g_{n+1}$ factors through $f_{n+1}$.

Now we can prove the main result of the section.

Proposition 5.14. $\overline{\mathbb{P} \mathcal{E}_{U_{1}}\left(V, U_{0}\right)}$ is a rational normal curve of degree $n$.

Proof. We consider the image of $\mathbb{P} \mathcal{E}_{1}=\mathbb{P} \overline{\mathcal{E}_{U_{1}}\left(V, U_{0}\right)}$ via the linear map

$$
\mathbb{P} F^{\prime \prime}: \mathbb{P} \operatorname{Ext}_{A}^{1}\left(V, U_{0}\right) \rightarrow \mathbb{P}\left(\mathcal{H}_{1}^{*} \otimes \mathcal{H}_{2}^{*} \otimes \ldots \otimes \mathcal{H}_{n}^{*} \otimes \operatorname{Ext}_{A}^{1}\left(V, U_{n}\right)\right) .
$$

We know that $\mathcal{E}_{n+1}$ is a linear subspace of dimension 1, by Lemma 5.13, Observe that

$$
\mathbb{P} F^{\prime \prime}\left(\mathbb{P} \mathcal{E}_{1}\right) \subseteq \mathbb{P}\left(\mathcal{H}_{1}^{*} \otimes \mathcal{H}_{2}^{*} \otimes \ldots \otimes \mathcal{H}_{n}^{*} \otimes \mathcal{E}_{n+1}\right)
$$

and the latter space is canonically isomorphic to $\mathbb{P}\left(\mathcal{H}_{1}^{*} \otimes \mathcal{H}_{2}^{*} \otimes \ldots \otimes \mathcal{H}_{n}^{*}\right)$. Moreover, the induced map

$$
\mathcal{F}: \mathbb{P} \mathcal{E}_{1} \rightarrow \mathbb{P}\left(\mathcal{H}_{1}^{*} \otimes \mathcal{H}_{2}^{*} \otimes \ldots \otimes \mathcal{H}_{n}^{*}\right)
$$

is the composition of

$$
\mathbb{P} \mathcal{E}_{1} \stackrel{\left(\mu_{1}, \mu_{2}, \ldots, \mu_{n}\right)^{T}}{\longrightarrow} \mathbb{P} \mathcal{H}_{1} \times \mathbb{P} \mathcal{H}_{2} \times \ldots \times \mathbb{P} \mathcal{H}_{n}
$$

followed by the canonical isomorphism

$$
\mathbb{P} \mathcal{H}_{1} \times \mathbb{P} \mathcal{H}_{2} \times \ldots \times \mathbb{P} \mathcal{H}_{n} \rightarrow \mathbb{P} \mathcal{H}_{1}^{*} \times \mathbb{P} \mathcal{H}_{2}^{*} \times \ldots \times \mathbb{P} \mathcal{H}_{n}^{*}
$$

and the Segre embedding

$$
\mathbb{P} \mathcal{H}_{1}^{*} \times \mathbb{P} \mathcal{H}_{2}^{*} \times \ldots \times \mathbb{P} \mathcal{H}_{n}^{*} \rightarrow \mathbb{P}\left(\mathcal{H}_{1}^{*} \otimes \mathcal{H}_{2}^{*} \otimes \ldots \otimes \mathcal{H}_{n}^{*}\right) .
$$

Since the maps $\mu_{i}$ are isomorphisms, then in appropriate bases $\mathcal{F}$ has the form

$$
(x: y) \mapsto\left(\ldots: x^{\varepsilon_{1}+\ldots+\varepsilon_{n}} y^{n-\varepsilon_{1}-\ldots-\varepsilon_{n}}: \ldots\right)_{\left(\varepsilon_{1}, \ldots, \varepsilon_{n}\right) \in\{0,1\}^{n}} .
$$

Thus $\mathcal{F}\left(\mathbb{P} \mathcal{E}_{1}\right)$ is the rational normal curve of degree $n$ (see for instance 8 , Ex.1.14]) and the same holds for $\mathbb{P} \mathcal{E}_{1}$. 


\section{Proof of Theorem 1.1}

Applying [5, Thm.5] we get that there is a short exact sequence

$$
\sigma: 0 \rightarrow U \rightarrow M \rightarrow V \rightarrow 0
$$

and $\operatorname{Sing}(M, N)=\operatorname{Sing}\left(\overline{\mathcal{E}_{M}(V, U)}, 0\right)$. The assumption $\operatorname{dim} \mathcal{O}_{M}-\operatorname{dim} \mathcal{O}_{N}=2$ means that

$$
2=[N, N]-[M, M]=\delta_{\sigma}(M)+\delta_{\sigma}^{\prime}(N)=\delta_{\sigma}(M)+\delta_{\sigma}^{\prime}(V) .
$$

If $\delta_{\sigma}(M)=0$ then $\operatorname{Sing}(M, N)=\operatorname{Reg}=\operatorname{Sing}\left(\mathcal{C}_{1}, 0\right)$, by [14, Cor.3.7]. Thus we may assume that $\delta_{\sigma}(M) \geq 1$. We know that $\delta_{\sigma}^{\prime}(V) \geq 1$, as the sequence $\sigma$ does not split. Altogether, we get that $\delta_{\sigma}(M)=\delta_{\sigma}^{\prime}(V)=1$. Hence the claim follows from Proposition 5.14 .

\section{Proof of Theorem 1.2}

Let $\left(\Gamma_{A}, \tau_{A}\right)$ denote the Auslander-Reiten quiver of the algebra $A$ (see [10] for details). We may identify the set of vertices of $\Gamma_{A}$ with the corresponding indecomposable modules. A connected component $\mathcal{C}$ of $\Gamma_{A}$ is called preprojective if it has no cyclic paths and any $\tau_{A}$-orbit in $\mathcal{C}$ contains a projective module (see [10]). Moreover, a module is said to be preprojective if it is isomorphic to a direct sum of modules from preprojective components.

Let $M$ and $N$ be preprojective modules such that $\operatorname{dim} \mathcal{O}_{M}-\operatorname{dim} \mathcal{O}_{N}=2$ and $\mathcal{O}_{N} \subseteq \overline{\mathcal{O}}_{M}$. By [14, Thm.1.1], we may assume that the modules $M$ and $N$ are disjoint. Moreover we may assume that $N$ is a direct sum of at most two indecomposable modules, by [14, Thm.1.2]. Repeating the proof of [14, Lem.6.3], we get that there are indecomposable direct summands $U$ and $V$ of $N$ such that

$$
[M, U]<[N, U],[U, M]=[U, N] \text { and }[M, V]=[N, V],[V, M]<[V, N] \text {. }
$$

Therefore $N$ is isomorphic to $U \oplus V$ and the claim follows from Theorem 1.1.

\section{Example}

Let $n \geq 3$ and $A$ be the path algebra of the quiver

$$
Q:
$$

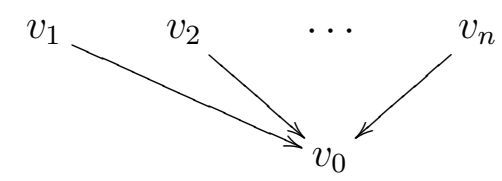


with $(n+1)$ vertices and $n$ arrows. We may replace modules by representations of the quiver $Q$, applying a Morita equivalence and 4].

Let $\left(a_{1}: b_{1}\right),\left(a_{2}: b_{2}\right), \ldots,\left(a_{n}: b_{n}\right)$ be pairwise different points of $\mathbb{P}^{1}$. We define four representations of $Q$ :
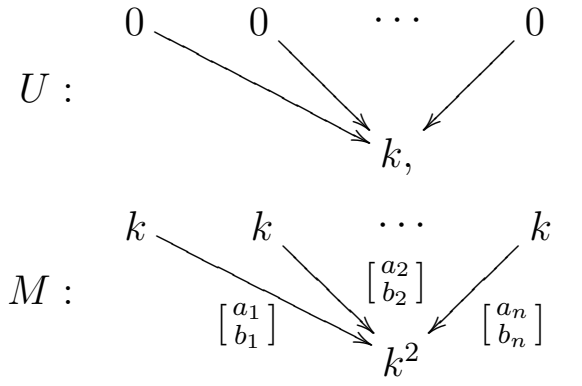

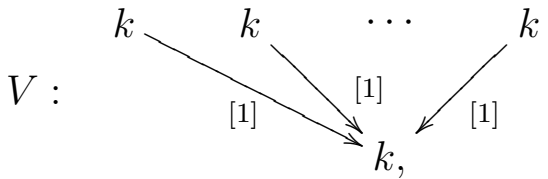

and $\quad N=U \oplus V$.

Then $\mathcal{S}_{V}(U, M) \simeq\left\{(a, b) \in k^{2} ; a_{i} b \neq b_{i} a\right.$ for $\left.i=1,2, \ldots, n\right\}$. In particular, $\mathcal{O}_{N} \subseteq \overline{\mathcal{O}}_{M}$, by Corollary 2.5. Since the representation $U$ is projective and the representation $V$ is injective, then

$$
[U, M]=[M, V]=2 \quad \text { and } \quad[U, U]=[U, V]=[V, V]=1 .
$$

Moreover, direct matrix calculations show that $[V, U]=0$ and $[M, M]=1$. Hence

$$
[U, M]=[U, N], \quad[M, V]=[N, V], \quad[N, N]-[M, M]=2,
$$

and we can apply Theorem 1.1. In fact, one can show that $\operatorname{Sing}(M, N)$ is the type of singularity of a cone over a rational normal curve of degree $(n-2)$. Observe that the representation $V$ is not preprojective (and $U$ is not preinjective) provided $n \geq 4$.

\section{References}

[1] M. Auslander, I. Reiten and S. Smalø, Representation Theory of Artin Algebras, Cambridge Studies in Advanced Mathematics 36 (1995).

[2] J. Bender and K. Bongartz, Minimal singularities in orbit closures of matrix pencils, Linear Algebra Appl. 365 (2003), 13-24.

[3] G. Bobiński and G. Zwara, Schubert varieties and representations of Dynkin quivers, Colloq. Math. 94 (2002), 285-309.

[4] K. Bongartz, A geometric version of the Morita equivalence, J. Algebra 139 (1991), 159-171. 
[5] K. Bongartz, Minimal singularities for representations of Dynkin quivers, Comment. Math. Helv. 63 (1994), 575-611.

[6] K. Bongartz, On degenerations and extensions of finite dimensional modules, Adv. Math. 121 (1996), 245-287.

[7] K. Bongartz and T. Fritzsche, On minimal disjoint degenerations for preprojective representations of quivers, Math. Comput. 72 (2003), 20132042.

[8] J. Harris, Algebraic geometry, a first course, GTM 133, Springer-Verlag (1992).

[9] W. Hesselink, Singularities in the nilpotent scheme of a classical group, Trans. Amer. Math. Soc. 222 (1976), 1-32.

[10] C. M. Ringel, Tame algebras and integral quadratic forms, Springer Lecture Notes in Math. 1099 (1984).

[11] G. Zwara, Degenerations of finite dimensional modules are given by extensions, Compos. Math. 121 (2000), 205-218.

[12] G. Zwara, Unibranch orbit closures in module varieties, Ann. Sci. École Norm. Sup. 35 (2002), 877-895.

[13] G. Zwara, Regularity in codimension one of orbit closures in module varieties, J. Algebra 283 (2005), 821-848.

[14] G. Zwara, Orbit closures for representations of Dynkin quivers are regular in codimension two, J. Math. Soc. Japan 57 (2005), 859-880.

Grzegorz Zwara

Faculty of Mathematics and Computer Science

Nicolaus Copernicus University

Chopina 12/18, 87-100 Toruń, Poland

E-mail: gzwara@mat.uni.torun.pl 